M. Rose \& D. Cass 
Network Working Group

Request for Comments: RFC 1006

Obsoletes: RFC 983

ISO Transport Service on top of the TCP

Version: 3
Marshall T. Rose, Dwight E. Cass Northrop Research and Technology Center

May 1987

Status of this Memo

This memo specifies a standard for the Internet community. Hosts on the Internet that choose to implement ISO transport services on top of the TCP are expected to adopt and implement this standard. TCP port 102 is reserved for hosts which implement this standard. Distribution of this memo is unlimited.

This memo specifies version 3 of the protocol and supersedes [RFC983]. Changes between the protocol as described in Request for Comments 983 and this memo are minor, but are unfortunately incompatible. 


\section{Introduction and Philosophy}

The Internet community has a well-developed, mature set of transport and internetwork protocols (TCP/IP), which are quite successful in offering network and transport services to end-users. The CCITT and the ISO have defined various session, presentation, and application recommendations which have been adopted by the international community and numerous vendors. To the largest extent possible, it is desirable to offer these higher level directly in the ARPA Internet, without disrupting existing facilities. This permits users to develop expertise with ISO and CCITT applications which previously were not available in the ARPA Internet. It also permits a more graceful convergence and transition strategy from TCP/IP-based networks to ISO-based networks in the medium-and long-term.

There are two basic approaches which can be taken when "porting" an ISO or CCITT application to a TCP/IP environment. One approach is to port each individual application separately, developing local protocols on top of the TCP. Although this is useful in the short-term (since special-purpose interfaces to the TCP can be developed quickly), it lacks generality.

A second approach is based on the observation that both the ARPA Internet protocol suite and the ISO protocol suite are both layered systems (though the former uses layering from a more pragmatic perspective). A key aspect of the layering principle is that of layer-independence. Although this section is redundant for most readers, a slight bit of background material is necessary to introduce this concept.

Externally, a layer is defined by two definitions:

a service-offered definition, which describes the services provided by the layer and the interfaces it provides to access those services; and,

a service-required definitions, which describes the services used by the layer and the interfaces it uses to access those services.

Collectively, all of the entities in the network which co-operate to provide the service are known as the service-provider. Individually, each of these entities is known as a service-peer.

Internally, a layer is defined by one definition:

a protocol definition, which describes the rules which each service-peer uses when communicating with other service-peers. 
Putting all this together, the service-provider uses the protocol and services from the layer below to offer the its service to the layer above. Protocol verification, for instance, deals with proving that this in fact happens (and is also a fertile field for many $\mathrm{Ph} . \mathrm{D}$. dissertations in computer science).

The concept of layer-independence quite simply is:

IF one preserves the services offered by the service-provider

THEN the service-user is completely naive with respect to the protocol which the service-peers use

For the purposes of this memo, we will use the layer-independence to define a Transport Service Access Point (TSAP) which appears to be identical to the services and interfaces offered by the ISO/CCITT TSAP (as defined in [ISO8072]), but we will in fact implement the ISO TPO protocol on top of TCP/IP (as defined in [RFC793, RFC791]), not on top of the the ISO/CCITT network protocol. Since the transport class 0 protocol is used over the TCP/IP connection, it achieves identical functionality as transport class 4. Hence, ISO/CCITT higher level layers (all session, presentation, and application entities) can operate fully without knowledge of the fact that they are running on a TCP/IP internetwork. 


\section{Motivation}

In migrating from the use of TCP/IP to the ISO protocols, there are several strategies that one might undertake. This memo was written with one particular strategy in mind.

The particular migration strategy which this memo uses is based on the notion of gatewaying between the TCP/IP and ISO protocol suites at the transport layer. There are two strong arguments for this approach:

1. Experience teaches us that it takes just as long to get good implementations of the lower level protocols as it takes to get implementations of the higher level ones. In particular, it has been observed that there is still a lot of work being done at the ISO network and transport layers. As a result, implementations of protocols above these layers are not being aggressively pursued. Thus, something must be done "now" to provide a medium in which the higher level protocols can be developed. Since TCP/IP is mature, and essentially provides identical

functionality, it is an ideal medium to support this development.

2. Implementation of gateways at the IP and ISO IP layers are probably not of general use in the long term. In effect, this would require each Internet host to support both TP 4 and TCP. As such, a better strategy is to implement a graceful migration path from TCP/IP to ISO protocols for the ARPA Internet when the ISO protocols have matured sufficiently.

Both of these arguments indicate that gatewaying should occur at or above the transport layer service access point. Further, the first argument suggests that the best approach is to perform the gatewaying exactly AT the transport service access point to maximize the number of ISO layers which can be developed.

NOTE: This memo does not intend to act as a migration or intercept document. It is intended ONLY to meet the needs discussed above. However, it would not be unexpected that the protocol described in this memo might form part of an overall transition plan. The description of such a plan however is COMPLETELY beyond the scope of this memo.

Finally, in general, building gateways between other layers in the TCP/IP and ISO protocol suites is problematic, at best.

To summarize: the primary motivation for the standard described in this memo is to facilitate the process of gaining experience with higher-level ISO protocols (session, presentation, and application). The stability and maturity of TCP/IP are ideal for 
providing solid transport services independent of actual implementation. 


\section{The Model}

The [ISO8072] standard describes the ISO transport service
definition, henceforth called TP.
ASIDE: This memo references the ISO specifications rather
than the CCITT recommendations. The differences
between these parallel standards are quite small,
and can be ignored, with respect to this memo,
without loss of generality. To provide the reader
with the relationships:
$\begin{aligned} \text { Transport service } \\ \text { Transport protocol [ISO8072] } \\ \text { Session protocol [ISO8073] } \\ {[\text { [ISO8327] }}\end{aligned}$

The ISO transport service definition describes the services offered by the TS-provider (transport service) and the interfaces used to access those services. This memo focuses on how the ARPA Transmission Control Protocol (TCP) [RFC793] can be used to offer the services and provide the interfaces.
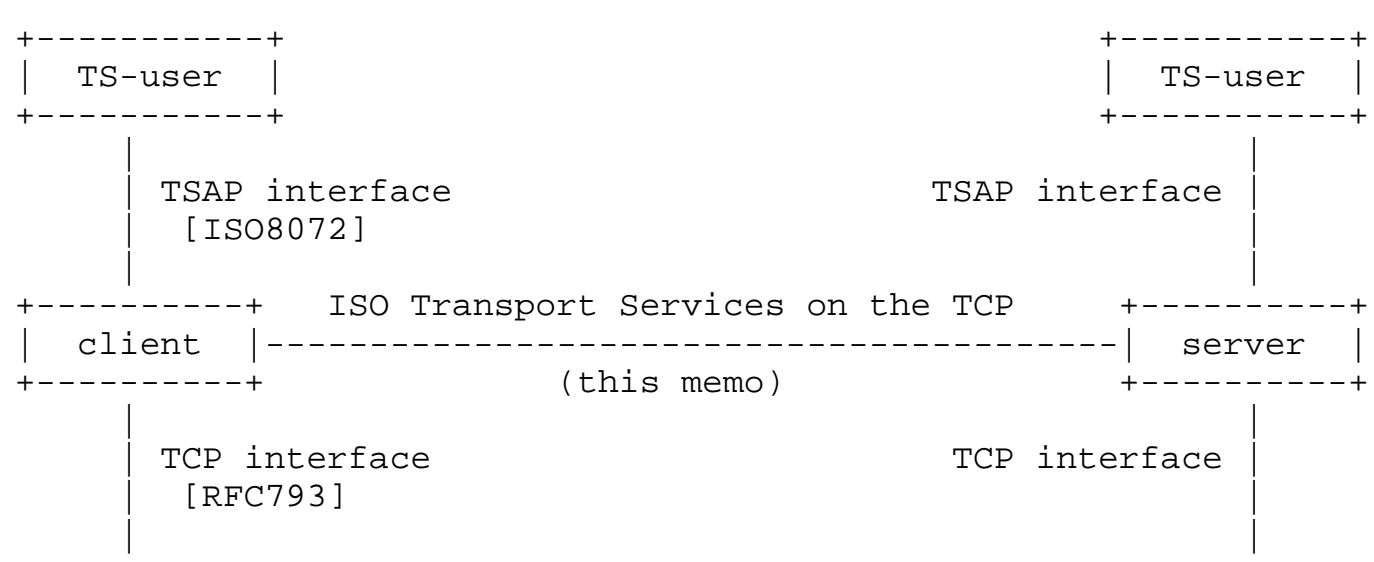

For expository purposes, the following abbreviations are used:

TS-peer a process which implements the protocol described by this memo

TS-user a process talking using the services of a TS-peer 
TS-provider the black-box entity implementing the protocol described by this memo

For the purposes of this memo, which describes version 2 of the TSAP protocol, all aspects of [ISO8072] are supported with one exception:

Quality of Service parameters

In the spirit of CCITT, this is left "for further study". A future version of the protocol will most likely support the QOS parameters for TP by mapping these onto various TCP parameters.

The ISO standards do not specify the format of a session port (termed a TSAP ID). This memo mandates the use of the GOSIP specification [GOSIP86] for the interpretation of this field. (Please refer to Section 5.2, entitled "UPPER LAYERS ADDRESSING".)

Finally, the ISO TSAP is fundamentally symmetric in behavior. There is no underlying client/server model. Instead of a server listening on a well-known port, when a connection is established, the TS-provider generates an INDICATION event which, presumably the TS-user catches and acts upon. Although this might be implemented by having a server "listen" by hanging on the INDICATION event, from the perspective of the ISO TSAP, all TSusers just sit around in the IDLE state until they either generate a REQUEST or accept an INDICATION. 
4. The Primitives

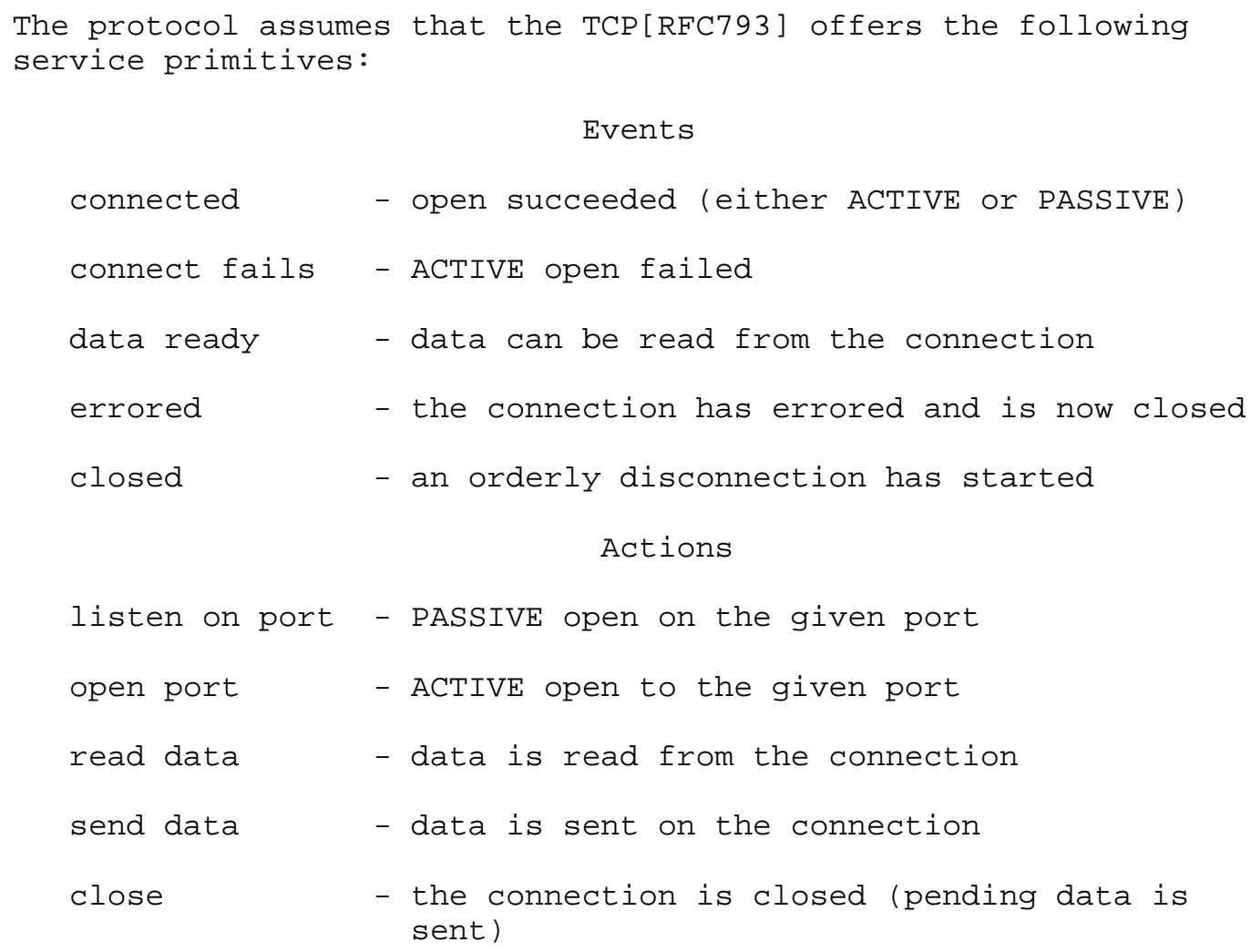

This memo describes how to use these services to emulate the following service primitives, which are required by [ISO8073]:

Events

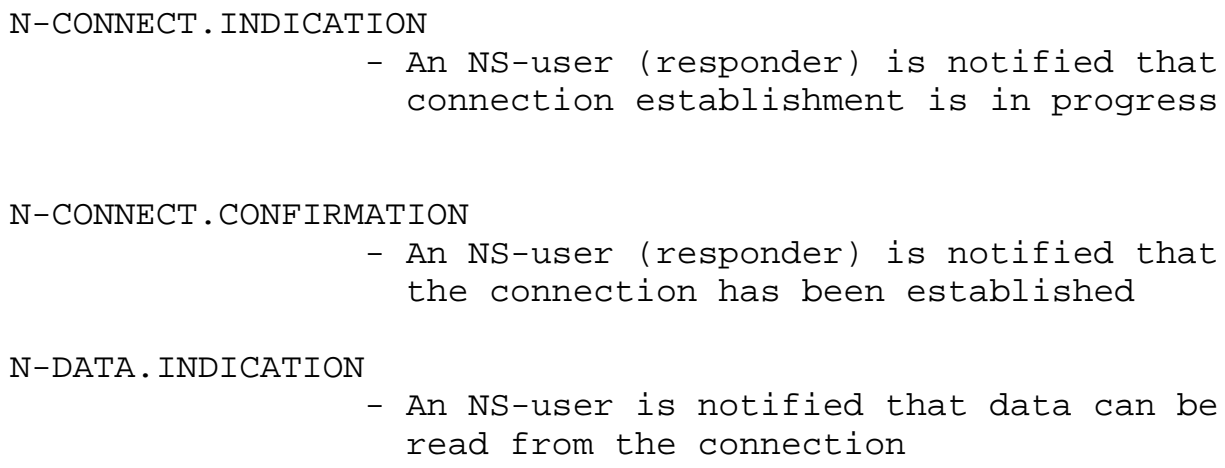

M. Rose \& D. Cass 


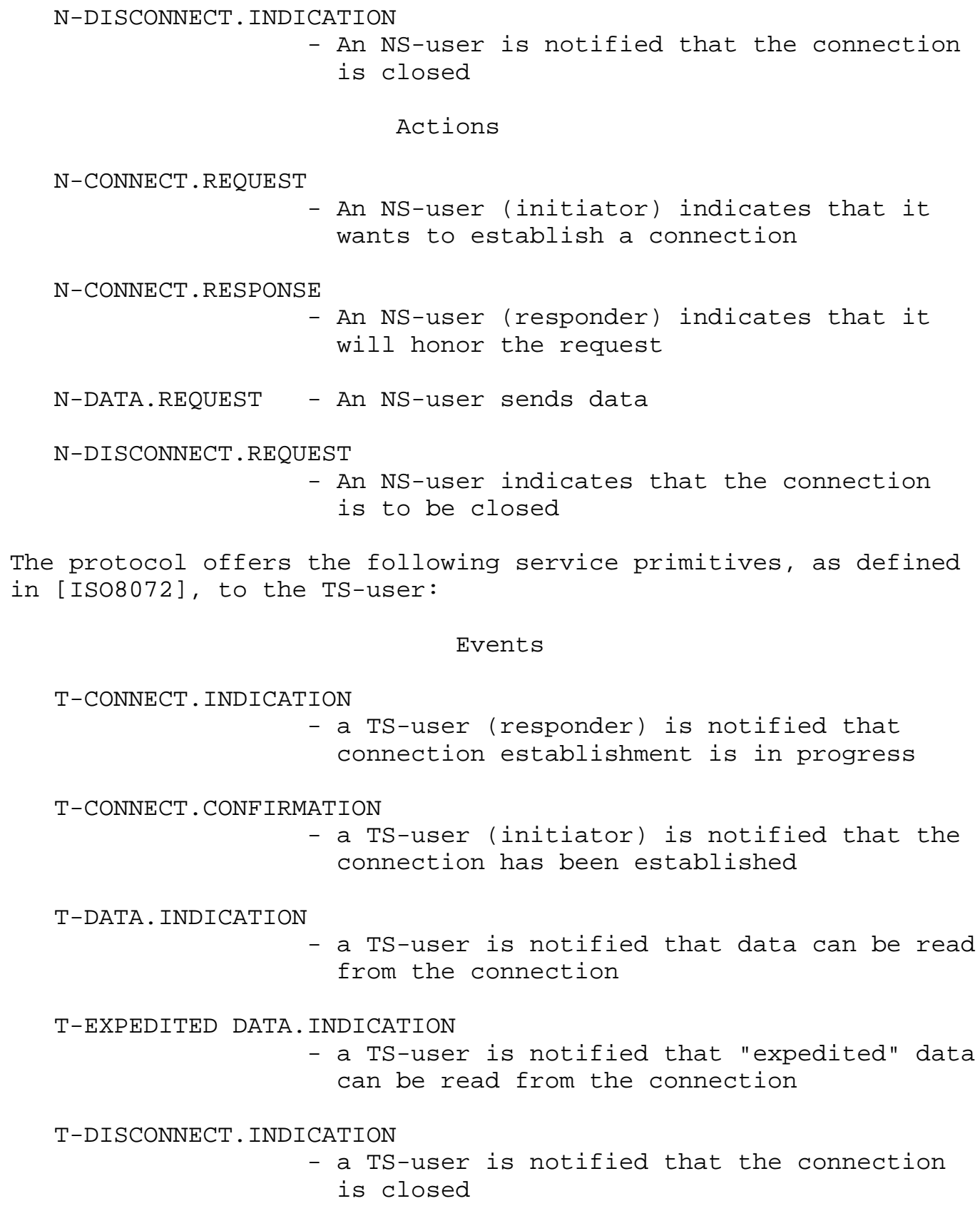




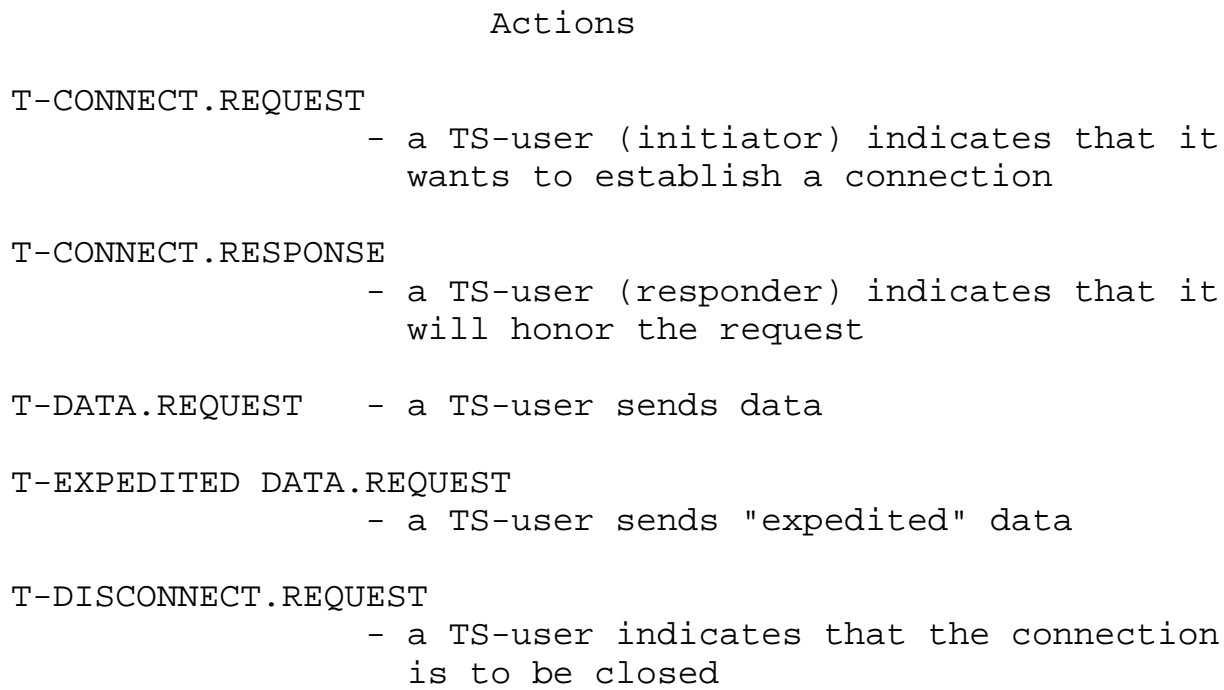




\section{The Protocol}

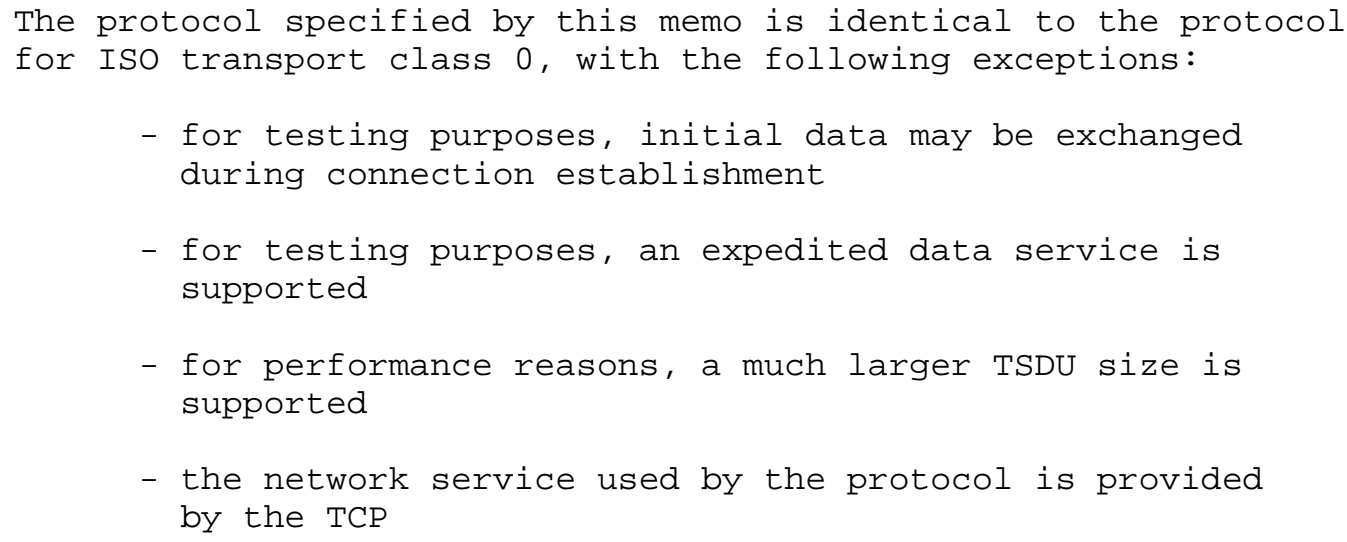

The ISO transport protocol exchanges information between peers in discrete units of information called transport protocol data units (TPDUs). The protocol defined in this memo encapsulates these TPDUs in discrete units called TPKTs. The structure of these TPKTs and their relationship to TPDUs are discussed in the next section.

PRIMITIVES

The mapping between the TCP service primitives and the service primitives expected by transport class 0 are quite straightforward:

\begin{tabular}{|c|c|}
\hline network service & $\mathrm{TCP}$ \\
\hline ---------------- & --- \\
\hline CONNECTION ESTABLISHMENT & \\
\hline $\mathrm{N}-\mathrm{CONNECT}$. REQUEST & open completes \\
\hline N-CONNECT.INDICATION & $\begin{array}{l}\text { listen (PASSIVE open) } \\
\text { finishes }\end{array}$ \\
\hline N-CONNECT . RESPONSE & listen completes \\
\hline N-CONNECT . CONF IRMATION & $\begin{array}{l}\text { open (ACTIVE open) } \\
\text { finishes }\end{array}$ \\
\hline DATA TRANSFER & \\
\hline N-DATA.REQUEST & send data \\
\hline N-DATA. INDICATION & data ready followed by \\
\hline
\end{tabular}


read data

\begin{tabular}{|c|c|c|}
\hline & CONNECTION RELEASE & \\
\hline & N-DISCONNECT . REQUEST & close \\
\hline & N-DISCONNECT. INDICATION & $\begin{array}{l}\text { connection closes or } \\
\text { errors }\end{array}$ \\
\hline Mapping & parameters is also straight-f & orward: \\
\hline & network service & TCP \\
\hline & ---------------- & --- \\
\hline & CONNECTION RELEASE & \\
\hline & Called address & $\begin{array}{l}\text { server's IP address } \\
(4 \text { octets })\end{array}$ \\
\hline & Calling address & $\begin{array}{l}\text { client's IP address } \\
(4 \text { octets) }\end{array}$ \\
\hline & all others & ignored \\
\hline & DATA TRANSFER & \\
\hline & NS-user data (NSDU) & data \\
\hline & CONNECTION RELEASE & \\
\hline
\end{tabular}

all parameters ignored

CONNECTION ESTABLISHMENT

The elements of procedure used during connection establishment are identical to those presented in [IS08073], with three exceptions.

In order to facilitate testing, the connection request and connection confirmation TPDUs may exchange initial user data, using the user data fields of these TPDUs.

In order to experiment with expedited data services, the connection request and connection confirmation TPDUs may negotiate the use of expedited data transfer using the negotiation mechanism specified in [ISO8073] is used (e.g., setting the "use of transport expedited data transfer service" bit in the "Additional Option selection" variable part). The default is not to use the transport expedited data transfer service.

M. Rose \& D. Cass

[Page 12] 
In order to achieve good performance, the default TPDU size is 65531 octets, instead of 128 octets. In order to negotiate a smaller (standard) TPDU size, the negotiation mechanism specified in [ISO8073] is used (e.g., setting the desired bit in the "TPDU Size" variable part).

To perform an N-CONNECT.REQUEST action, the TS-peer performs an active open to the desired IP address using TCP port 102 . When the TCP signals either success or failure, this results in an N-CONNECT. INDICATION action.

To await an N-CONNECT.INDICATION event, a server listens on TCP port 102. When a client successfully connects to this port, the event occurs, and an implicit N-CONNECT.RESPONSE action is performed.

NOTE: In most implementations, a single server will perpetually LISTEN on port 102, handing off connections as they are made

\section{DATA TRANSEER}

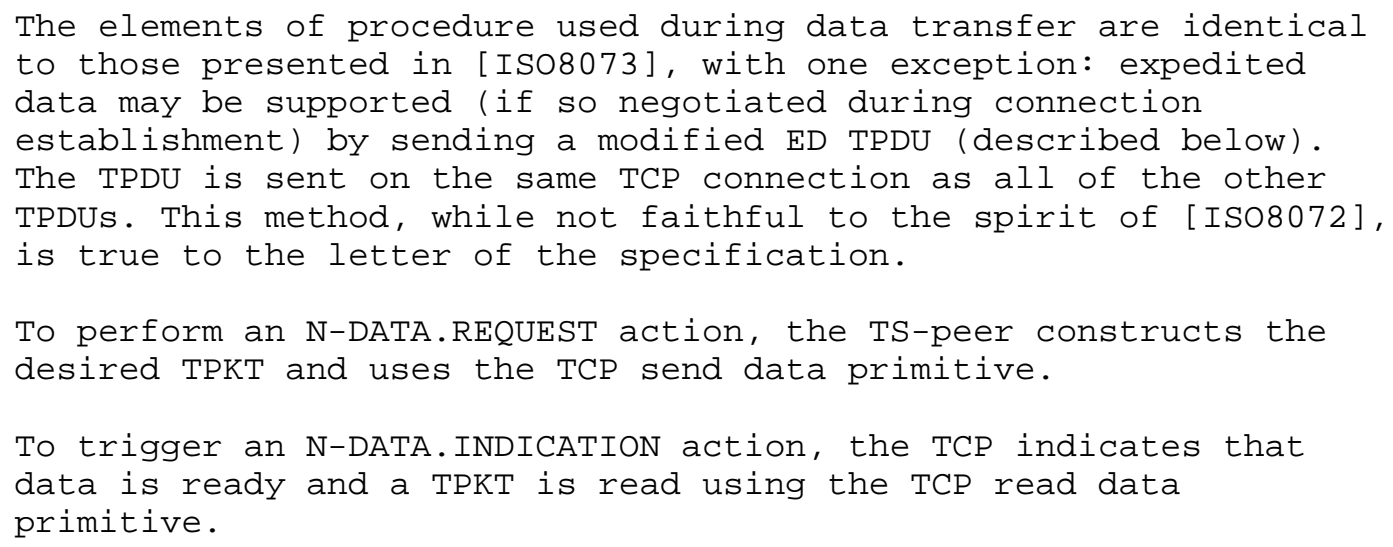

CONNECTION RELEASE

To perform an N-DISCONNECT.REQUEST action, the TS-peer simply closes the TCP connection.

If the TCP informs the TS-peer that the connection has been closed or has errored, this indicates an N-DISCONNECT. INDICATION event. 


\section{Packet Format}

A fundamental difference between the TCP and the network service expected by TPO is that the TCP manages a continuous stream of octets, with no explicit boundaries. The TPO expects information to be sent and delivered in discrete objects termed network service data units (NSDUs). Although other classes of transport may combine more than one TPDU inside a single NSDU, transport class 0 does not use this facility. Hence, an NSDU is identical to a TPDU for the purposes of our discussion.

The protocol described by this memo uses a simple packetization scheme in order to delimit TPDUs. Each packet, termed a TPKT, is viewed as an object composed of an integral number of octets, of variable length.

NOTE: For the purposes of presentation, these objects are shown as being 4 octets ( 32 bits wide). This representation is an artifact of the style of this memo and should not be interpreted as requiring that a TPKT be a multiple of 4 octets in length.

A TPKT consists of two parts: a packet-header and a TPDU. The format of the header is constant regardless of the type of packet. The format of the packet-header is as follows:

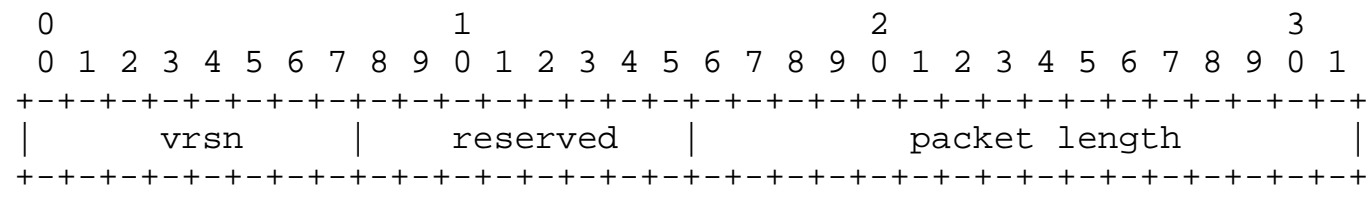

where:

vrsn 8 bits

This field is always 3 for the version of the protocol described in this memo.

packet length 16 bits (min=7, $\max =65535)$

This field contains the length of entire packet in octets, including packet-header. This permits a maximum TPDU size of 65531 octets. Based on the size of the data transfer (DT) TPDU, this permits a maximum TSDU size of 65524 octets.

The format of the TPDU is defined in [ISO8073]. Note that only TPDUs formatted for transport class 0 are exchanged (different transport classes may use slightly different formats). 
To support expedited data, a non-standard TPDU, for expedited data is permitted. The format used for the ED TPDU is nearly identical to the format for the normal data, DT, TPDU. The only difference is that the value used for the TPDU'S code is ED, not DT:

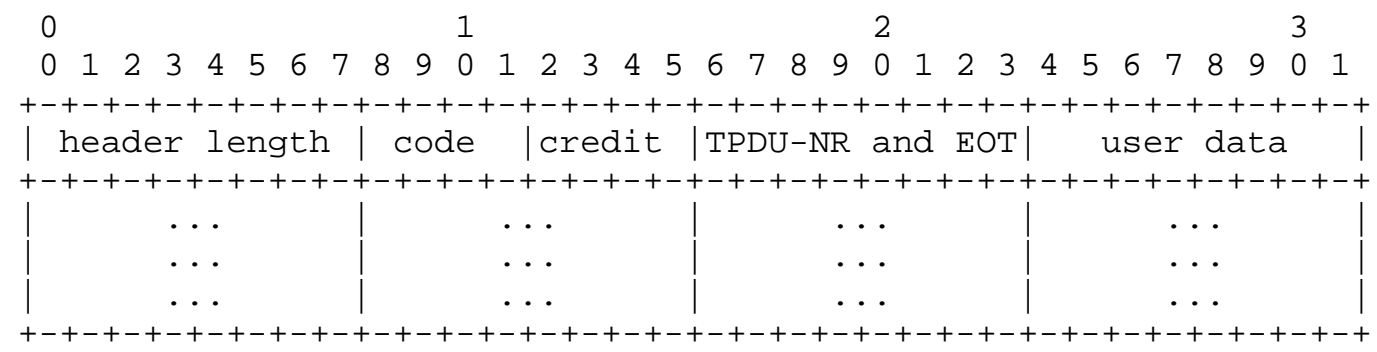

After the credit field (which is always ZERO on output and ignored on input), there is one additional field prior to the user data.

TPDU-NR and EOT 8 bits

Bit 7 (the high-order bit, bit mask 1000 0000) indicates the end of a TSDU. All other bits should be ZERO on output and ignored on input.

Note that the TP specification limits the size of an expedited transport service data unit (XSDU) to 16 octets. 


\section{Comments}

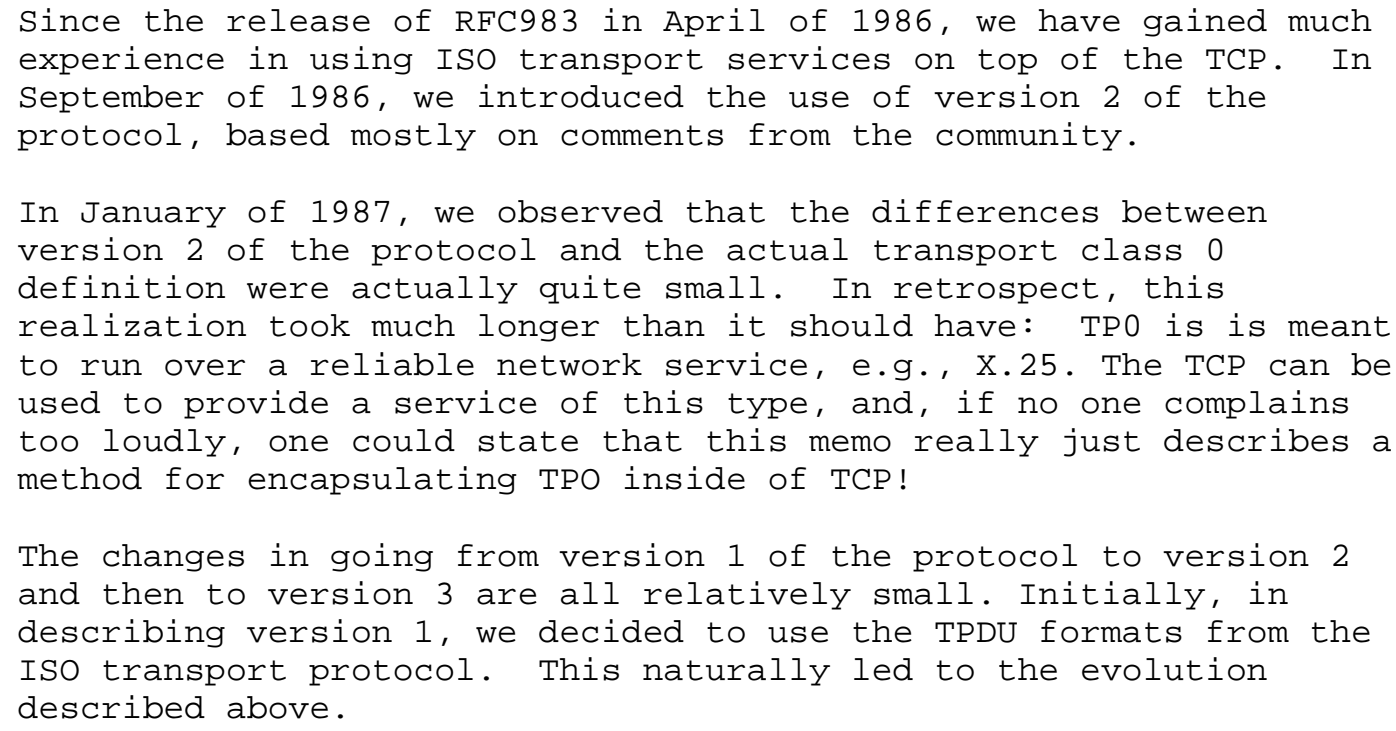


8. References

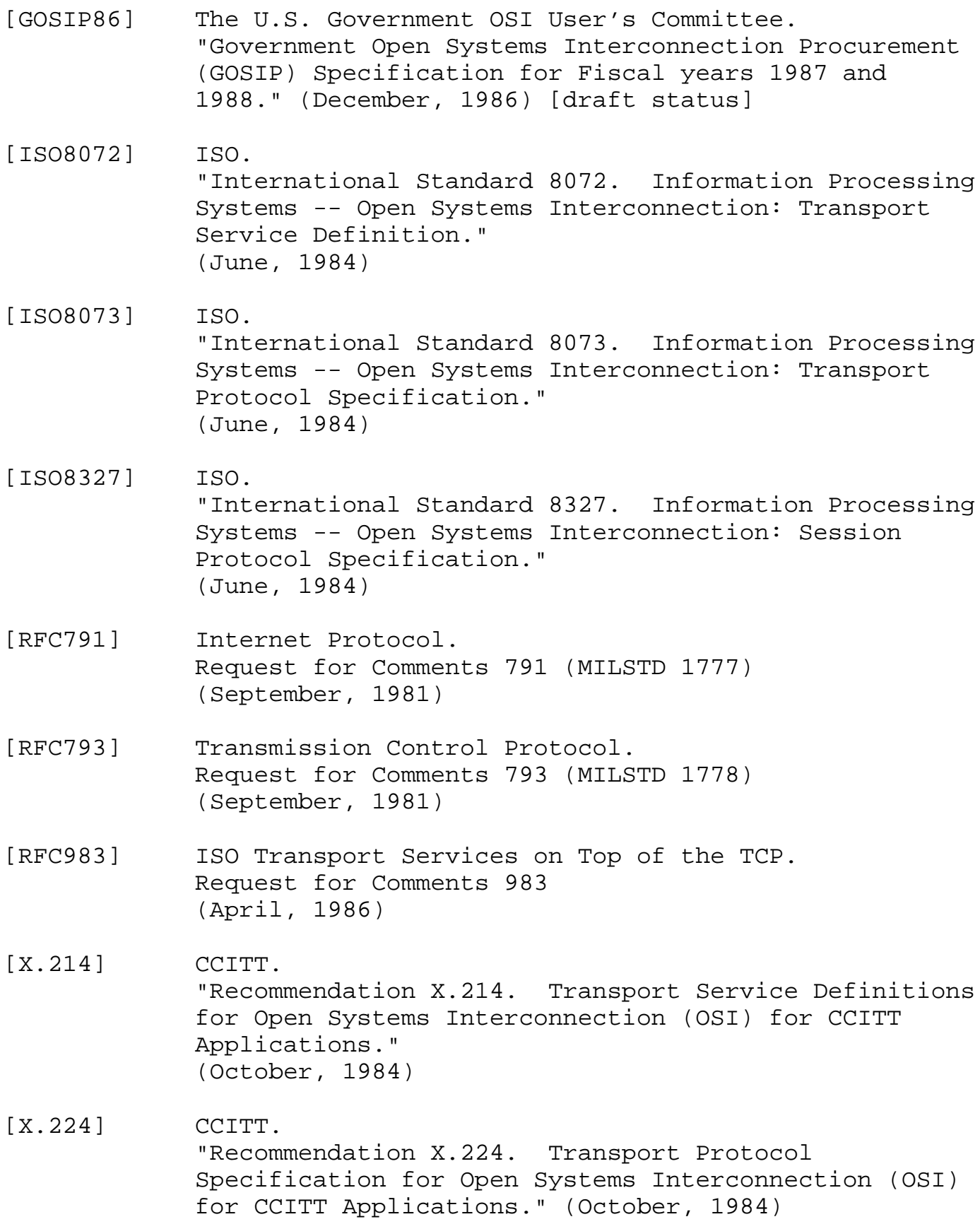


$[\mathrm{X} .225]$

CCITT.

"Recommendation X.225. Session Protocol Specification

for Open Systems Interconnection (OSI) for CCITT

Applications."

(October, 1984) 